\title{
Network pharmacology-based analysis of the mechanisms of Tripterygium wilfordii Hook F on cardiovascular disease
}

\author{
Bingwu Huang ${ }^{1}$, Chengbin Huang ${ }^{2}$, Liuyan Zhu ${ }^{3}$, Lina Xie ${ }^{4}$, Yi Wang ${ }^{5}$, and Ning Zhu ${ }^{5}$ \\ ${ }^{1}$ The Second Affiliated Hospital and Yuying Children's Hospital of Wenzhou Medical \\ University, Department of Anesthesiology \\ ${ }^{2}$ The Second Affiliated Hospital and Yuying Children's Hospital of Wenzhou Medical \\ University, Department of Orthopedic Surgery \\ ${ }^{3}$ The Wenzhou Third Clinical Institute Affiliated To Wenzhou Medical University, \\ Wenzhou People's Hospital, Department of General Practice \\ ${ }^{4}$ The Wenzhou Third Clinical Institute Affiliated To Wenzhou Medical University, \\ Wenzhou People's Hospital, Department of Neurosurgery \\ ${ }^{5}$ The Wenzhou Third Clinical Institute Affiliated To Wenzhou Medical University, \\ Wenzhou People's Hospital
}

September 28, 2020

\begin{abstract}
Background and purpose: TwHF has been used in traditional Chinese medicines for treating CVD. However, the underlying pharmacological mechanisms of the effects of TwHF against CVD remain to be elucidated. The aim of the present study is to reveal the pharmacological mechanisms of TwHF acting on CVD based on a pharmacology approach. Experimental approach: The active compounds were screened by TCMSP according to ADME. The potential targets of TwHF were predicted by SwissTargetPrediction database. The CVD-related therapeutic targets were obtained by the DrugBank, the OMIM database and the GeneCards database. PPI network was constructed by STRING database. GO and KEGG pathway enrichment analyses were performed by R package. The network of drug-targets-diseases-pathways was constructed by Cytoscape software. Key results: A total of 51 effective ingredients of TwHF and the 178 common targets of TwHF and CVD-related were collected. AKT1, APP, MAPK, PIK3CA and TP53 was identified the core targets involved in the action of TwHF on CVD. Top ten GO and KEGG pathways were identified with a P value [?] 0.01. Finally, we constructed the network of TwHF-targets-CVD-GOKEGG. Conclusion and implications: Our results demonstrated that the main active compound of TwHF exerts cardiovascular protective effects and the core targets and pathways associated with the effects of TwHF on CVD. By the construction of the network of TwHF-targets-CVD-GO-KEGG, network pharmacology uncovered the pharmacological mechanisms of the action of TwHF on CVD and indicated a novel perspective to identify the intricate interactions among TwHF, candidate targets and related pathways.
\end{abstract}

\begin{abstract}
Background and purpose: Tripterygium wilfordii Hook F (TwHF) has been used in traditional Chinese medicines for treating cardiovascular disease (CVD). However, the underlying pharmacological mechanisms of the effects of TwHF against CVD remain to be elucidated. The aim of the present study is to reveal the pharmacological mechanisms of TwHF acting on CVD based on a pharmacology approach.
\end{abstract}

Experimental approach: The active compounds were screened by Traditional Chinese Medicine Systems Pharmacology Database (TCMSP) according to the absorption, distribution, metabolism, and ex- 
cretion (ADME). The potential targets of TwHF were predicted by SwissTargetPrediction database. The CVD-related therapeutic targets were obtained by the DrugBank, the OMIM database and the GeneCards database. Protein-protein interaction (PPI) network was constructed by STRING database. Gene ontology (GO) and Kyoto Encyclopedia of Genes and Genomes (KEGG) pathway enrichment analyses were performed by $\mathrm{R}$ package. The network of drug-targets-diseases-pathways was constructed by Cytoscape software.

Key results: A total of 51 effective ingredients of TwHF and the 178 common targets of TwHF and CVD-related were collected. AKT1, amyloid precursor protein (APP), Mitogen-activated protein kinase 1 (MAPK), phosphatidylinositol 3-kinase catalytic subunit alpha (PIK3CA) and cellular tumor antigen p53 (TP53) was identified the core targets involved in the action of TwHF on CVD. Top ten GO (biological processes, cellular components and molecular functions) and KEGG pathways were identified with a $\mathrm{P}$ value [?] 0.01. Finally, we constructed the network of TwHF-targets-CVD-GO-KEGG.

Conclusion and implications: Our results demonstrated that the main active compound of TwHF exerts cardiovascular protective effects and the core targets and pathways associated with the effects of TwHF on CVD. By the construction of the network of TwHF-targets-CVD-GO-KEGG, network pharmacology uncovered the pharmacological mechanisms of the action of TwHF on CVD and indicated a novel perspective to identify the intricate interactions among TwHF, candidate targets and related pathways.

\section{Introduction}

Cardiovascular disease (CVD) is a collective term for cardiovascular and cerebrovascular diseases, which is the first cause of death in the world[1]. The burden of CVD is on the rise globally, especially for low and middle income countries (LMIC)[2, 3]. In 2013, the World Health Organization (WHO) launched the $25 \times 25$ Global Action Plan, an ambitious road map for countries to reduce premature mortality that related to noncommunicable diseases (CVD, cancer, diabetes mellitus, and chronic respiratory diseases, etc.) by $25 \%$ by $2025[4]$. Although Western medicine have made good progress in reducing the risk of cardiovascular events and total mortality, patients with long-term cardiovascular treatment still have tradeoffs in adherence that might lead to discontinuation of these drugs. This can be attributed to the adverse reactions caused by multiple pharmacologic agents and some drugs that beyond the affordability of LMIC[3, 5]. Therefore, strategies that develop new drugs are urgently needed for CVD therapies. Traditional Chinese medicine (TCM) has evolved over thousands of years and has gained widespread clinical applications. Chinese, especially elders, has a special place in their heart for Traditional Chinese Medicine (TCM). As a critical component of complementary and alternative medicine, TCM medications has been used for primary and secondary prevention of CVD[6].

Tripterygium wilfordii Hook F (TwHF), also known as Leigongteng and Thunder God Vine in traditional Chinese medicine (TCM), has possesses a variety of pharmacological activities such as anti-cancer, anti-inflammation, anti-fibrosis, anti-atherosclerosis and anti-autoimmune disorders[7-9]. Recently, several fundamental researches have indicated that low-dose TwHF can prevent cardiovascular diseases. Low-dose TwHF can improve the inflammatory reaction, reduce myocardial injury, and optimize acute coronary syndrome (ACS) rat's condition with inhibition of myocardial apoptosis[10]. TwHF extracts were shown to have cardioprotection effects by inducing the activation of Nrf2/HO-1 defense pathway, inhibiting the activation of NF-KB pathway and reducing the expression of NLRP3 inflammasome[11-13]. In addition, extracts can not only improve the vascular function in atherosclerosis, but may also aid in the prevention of in-stent restenosis formation following endovascular treatment of lower-extremity artery disease[14, 15]. However, a systematical understanding of how the multiple therapeutic targets work together to exert therapeutic effects on CVD has not been fully elucidated.

Network pharmacology is an innovative method to analyze the complex relationship between drug and disease at the system level, which can provide clues for discovering new drug[16]. This approach integrates and constructs the complex networks among drug targets, disease targets, and biological processes[17]. With the help of network pharmacology, it is possible to reveal potential drug-target-disease interactions and realize novel therapeutic application beyond the traditional TCM application[18]. In this paper, pharmacokinetic 
evaluation, target prediction, network, and pathway analysis using multiple available public databases and bioinformatics resources, have systematically elucidated the mechanisms of therapeutic effects of TwHF on CVD.

\section{Materials and Methods}

\subsection{Active Components Screening}

Traditional Chinese Medicine Systems Pharmacology Database (TCMSP, https://tcmspw.com/tcmsp.php) is an efficient systems pharmacology platform, which can be used to assess the pharmacokinetics of TCMs or related compounds[19]. It can provide information on the absorption, distribution, metabolism, and excretion (ADME) properties of compounds, such as oral bioavailability (OB), drug likeness (DL), Caco-2 permeability (Caco-2), blood-brain barrier (BBB), and so on. OB represents to the speed and degree of absorbing drugs into the circulatory system, which is a reliable indicator to evaluate the intrinsic quality of drugs objectively. DL is calculated by comparing the functional or physical properties of compounds with those of the majority of known drugs, which refers to the sum of the pharmacokinetic properties and safety[20]. In present study, the compound name "leigongteng" was entered to the search box and active ingredients with $\mathrm{OB} \geq 30 \%$ and $\mathrm{DL} \geq 0.18$ were selected as candidate components for subsequent analysis. In addition, the two/three dimensional (2D/3D) structure, canonical SMILES, and PubChem ID of candidate components were prepared and calibrated using the Traditional Chinese Medicines Integrated Database (TCMID,http://www.megabionet.org/tcmid/)[21] and the PubChem (https://pubchem.ncbi.nlm.nih.gov/) database[22].

\subsection{Targets fishing}

\subsubsection{Identified and Predicted Targets of TwHF}

The target of active components in TwHF were collected from the SwissTargetPrediction (http://www.swisstargetprediction.ch), which is a free public resource designed to accurately predict targets for bioactive molecules[23]. Potential therapeutic targets were predicted by inputting these components SMILES into SMILES string (s) and searching for their similar molecules. The screening condition was limited to "Homo sapiens" and high probability targets (probability $\mathrm{P}<0.05$ ) were collected after duplicate contents were eliminated.

\subsubsection{Target Identification of Known Therapeutic Targets Acting on CVD}

The CVD-related therapeutic targets were found from the DrugBank (http://www.drugbank.ca)[24], the OMIM database (https://omim.org)[25], and the GeneCards database (https://www.genecards.org)[26]. DrugBank is a freely available network database, which provides molecular information about drugs, drug targets, drug effects, and drug interactions. OMIM database, a comprehensive web resource, is focusing on genes, genetic phenotypes, and their relationships. In addition, GeneCards is an integrative database that provides comprehensive information on all annotated and predicted human genes. The query "Cardiovascular disease" was used as the keyword to search for CVD-related targets among three databases.

\subsection{Protein-Protein Interaction (PPI) Network Construction and Analysis}

The identified targets were uploaded to the STRING database v11.0 (http://string-db.org)[27] to obtain the protein-protein interaction information, including the physical and functional associations. The protein interactions were limited to confidence score of 0.9 or higher. The core target genes were determined based on criterion of the highest interactive scores and the most interaction.

\subsection{GO and KEGG Pathway Enrichment Analyses}

GO analysis can supplies evidence-supported annotations to describe gene product biological functions, including biological pathways, cellular components, and molecular functions[28]. KEGG analysis can give functional meaning to genes or genomes at molecular and higher levels[29]. Enrichment analyses of GO of core target genes and KEGG were performed using R (version 3.6.0 for Windows), including biological 
function (BP), cellular component (CC), and molecular function (MF). By using a cut-off value adjusted to $\mathrm{P}<0.05$, top ten GO enrichments and KEGG pathways were screened.

\subsection{Construction of network relationships}

Cytoscape is an open source software project, which can integrate biomolecular interaction networks with high-throughput expression data and other molecular states into a versatile and interactive visualization framework[30]. The core targets of TwHF against CVD were constructed for KEGG-GO enrichment visualization by Cytoscape (v3.7.1) software[31]. In interactive network, nodes represent components, targets, $\mathrm{GO}$ and pathways, and edges represent the interaction of each other.

\subsection{Molecular Docking}

In addition, the crystal structures of target proteins were collected from the Protein Data Bank (http: //www.pdb.org/) and decorated by removing the ligands and water motifs, adding hydrogen, and optimizing the mutation sites by the Pymol (version 2.3). The 3D chemical structural formulas of candidate compounds were collected from PubChem and energy minimizing by using ChemBioDraw 3D (version 14). The binding ability, sites, and interactions between compounds and targets were analyzed by Pymol, AutoDockTools (version 1.5.6), and Discovery Studio 2020 Client[32, 33]. Autodock vina (1.1.2) was used to conducte docking between compounds and target proteins.

\section{Results}

\subsection{Active compounds screening}

A total of 51 effective ingredients of TwHF that satisfied OB $\geq 30 \%$ and DL $\geq 0.18$ were selected from TCMSP. Among them, only 41 candidate compounds have the two dimensional (2D) structure, canonical SMILES, and PubChem ID (Table 1).

\subsection{Targets Identification of TwHF and CVD.}

In total, 827 candidate targets for TwHF were identified using SwissTargetPrediction (Supplementary Table). 76 known CVD targets were obtained from the DrugBank database, 358 known CVD targets were collected from the GeneCards database, and 474 known CVD-related targets were found from the OMIM database (Supplementary Table). Then, 802 known CVD targets were identified by eliminating the repeated CVDrelated targets. Finally, we compared the targets of CVD and TwHF, and the 178 same targets were collected for subsequent analysis (Supplementary Table).

\subsection{PPI Network Construction and Analysis}

A total of 178 identified targets were uploaded to the STRING database to identify the functional partnerships and interactions between them (Figure 2). A total of 178 gene entries with prioritized interactive scores and were screened, which served as the key putative targets involved in the effects of TwHF on CVD. AKT1, amyloid precursor protein (APP), Mitogen-activated protein kinase 1 (MAPK), phosphatidylinositol 3-kinase catalytic subunit alpha (PIK3CA) and cellular tumor antigen p53 (TP53) was identified according to the highest interactive scores and the most interaction.

\subsection{GO and KEGG Pathway Enrichment Analyses}

The major targets could be categorized into various functional modules by Gene Ontology enrichment analysis. An introduction of the GO analysis was discovered with the enriched conditions in the biological process (BP), cellular component (CC), and molecular function categories (MF) (Figure 3). Depending on the outcomes of GO enrichment, the enriched biological process categories were dominated by ERBB signaling pathway, regulation of generation of precursor metabolites and energy, peptidyl-serine phosphorylation, aging, peptidyl-serine modification, regulation of developmental growth, neuron death, regulation of DNA metabolic process, cellular response to peptide, and response to oxidative stress. Cell component analysis showed that spindle mainly accounted for the largest proportion. The enriched molecular function categories were dominated by phosphatase binding and protein serine/threonine kinase activity. 
The KEGG pathway analysis showed that 178 targets were associated with cancer, melanoma, platinum drug resistance, glioma, chronic myeloid leukemia, endocrine resistance, sphingolipid signaling pathway, neurotrophin signaling pathway, thyroid hormone signaling pathway, apoptosis, cellular senescence, hepatitis $\mathrm{C}$, and hepatitis B (Figure 4).

\subsection{Construction of network}

The network visualization of TwHF-targets-CVD-GO-KEGG were generated by using Cytoscape software (Figure 5).

\subsection{Molecular Docking}

The crystal structures of potential targets, including AKT1 (PDB: 6CCY), APP (PDB:5BUO), MAPK1 (PDB:6SIG), PIK3CA (PDB:4TTU) and TP53 (PDB: 6RZ3) were collected from the RCSB Protein Data Bank. Figure 5 showed celaxanthin binds to AKT1 with a binding pocket consisting of SER-240 (2.9 A); hypodiolide A fails to bind to APP without a binding pocket; triptofordin B2 binds to MAPK1 with a binding pocket consisting of SER-153 (3.3 A) and ARG-155 (3.3 A); triptofordin B2 binds to PIK3CA with a binding pocket consisting of GLN-582 $(3.1 \mathrm{~A})$; Celallocinnine binds to TP53 with a binding pocket consisting of LEU-111 (3.2 and 3.0 A), ASN-131 (3.1 A) and TYR-126 (2.9 A).

\section{Discussion}

There is an urgent need to develop new strategies for CVD treatment because of the fast growing burden of CVD and the poor efficacy and side effects of the existing approved medicines. Network pharmacology has been used for of interaction between drugs and targets of diseases, and it is capable of comprehensively describing complexities among drugs and diseases[33, 34]. Therefore, the development of network pharmacology techniques that can predict multiple drug-target interactions may hold the key to future drug discoveries in complex diseases such as CVD. TwHF exhibits therapeutic efficacy in preclinical models of CVD has been identified in several studies[35-37]. In this study, the underlying mechanism of protective effects of TwHF on CVD was uncovered by a network pharmacology strategy. Therapeutic targets and the signaling pathways in which they participate were investigated by databases screening, PPI network construction, and pathway enrichment analysis. Furthermore, molecular docking was applied to validate the specific interactions between core targets and CVD.

In this study, 51 active compounds of TwHF were determined based on ADME. Pharmacological analysis suggested that these active components may have protective effects on CVD. Nobiletin has been reported to in H9c2 cardiomyocytes attenuate hypoxia/reoxygenation injury by the inhibition of oxidative stress and apoptosis as well as myocardial ischemia and reperfusion injury in vivo[38, 39]. Triptonide ameliorates diabetic cardiomyopathy via mediating inflammation[40, 41]. Isoxanthohumol regulate vivo vascular proliferation in vivo the -inflammatory crosstalk of vascular cells, contributing to the treatment of angiogenesis and inflammation-related diseases[42]. Stigmasterol protects against Ang II-induced aortic smooth muscle cell proliferation by the arrest of the cell-cycle, promotion of apoptosis and ROS production[43]. Kaempferol attenuates cardiac hypertrophy and isoproterenol-induced heart failure in diabetic rats[44, 45].

Subsequently, targets of TwHF and CVD were also identified. 178 common targets between TwHF and CVD were selected. Finally, we collected 5 cores candidate genes for further analysis. The interactive values and interaction indicate that these targets are tightly contact with other targets in "CVD-target PPI network" and responsible for TwHF actiong on CVD and pathogenesis of CVD. The alterations of Akt signaling play an important role in many cardiovascular pathological processes such as atherosclerosis, cardiac hypertrophy, and vascular remodeling. Several Akt inhibitors have been developed and tested as anti-tumor agents. They could be potential novel therapeutics for the cardiovascular diseases[46]. PIK3R1, MAPK1 and PIK3CA may modulate platelet activation and be involved in CVD[47]. Class I phosphatidylinositol 3-kinases (PI3Ks) are heterodimeric molecules composed of a regulatory subunit (usually p85 regulatory subunit) and a catalytic subunit (usually p110 catalytic subunit)[48, 49]. The catalytic subunit p110 $\alpha$ of PI3K is encoded by the gene PIK3CA, which regulates doxorubicin-induced biventricular atrophy and remodeling in right 
ventricular dysfunction[50]. Indeed, the compounded cardiovascular risk of PI3K $\alpha$ inhibitor use in breast cancer, is particularly relevant given the prevalence of $\mathrm{p} 110 \alpha$ gain-of-function mutations[51]. MAPK kinases including p38 mitogen activated protein kinase (p38), extracellular signal-regulated kinase1/2 (ERK), and c-Jun NH2 terminal protein kinase (JNK), are major components of pathways controlling embryogenesis, cell differentiation, cell proliferation, and cell death[52]. The inhibition of the phosphorylation of JNK, p38MAPK, and ERK1/2 could block proliferation and migration of vascular smooth muscle cells[53-55]. APP is associated with platelet adhesion to amyloid peptides and potentiation of thrombus formation[56, 57]. It was reported TP53 can differentiate patient with left main coronary artery disease (CAD) from patients healthy participants[58].

Top ten GO of each category (BP, MF, CC) and KEGG pathway associated with TwHF acting on CVD were classified. The results indicated that the major hubs were significantly involved in multiple BPs, including ERBB signaling pathway, regulation of generation of precursor metabolites and energy, peptidylserine phosphorylation, aging, peptidyl-serine modification, regulation of developmental growth, neuron death, regulation of DNA metabolic process, cellular response to peptide, and response to oxidative stress. KEGG pathway enrichments analysis showed TwHF may impart therapeutic effects on CVD mainly through cancer pathways. Cancer and cardiovascular disease (CVD) share overlapping pathophysiology and risk factors as well as biological mechanisms[59]. Protein-protein interactions analysis have varied roles in driving and maintaining the growth of cancer and $\operatorname{CVD}[60,61]$. TwHF was also identified for the treatment of multiple cancer[62-64].

According to screening criteria of high OB, Celaxanthin, Hypodiolide A, Triptofordin B2, Triptofordin B2, Celallocinnine were chosen for the compound-ligand interaction analysis by molecular docking to explore the anti-CVD effects of TwHF. The results molecular docking reflected that these active compounds possess suitable anti-CVD activity. However, the active properties TwHF and the molecular targets of CVD need to be further verified in future studies.

\section{Conclusion}

In summary, the network pharmacology approach was performed to unveil the chemical basis and investigate the action mechanism of TwHF on CVD. 51 active compounds of TwHF and 5 core target genes of TwHF against CVD, including AKT1, APP, MAPK, PIK3CA and TP53 were identified. Based on the analysis of GO and KEGG, cancer pathway was found to be closely associated with the protective effect of TwHF on CVD. The present study provides a theoretical basis and a clue for the pharmacological mechanism study of TwHF on CVD. However, further experimental studies of these prediction results are needed to validate potential application.

1. Olvera Lopez E, Ballard BD, Jan A: Cardiovascular Disease . In: StatPearls. Treasure Island (FL): StatPearls Publishing

Copyright (C) 2020, StatPearls Publishing LLC.; 2020.

2. Dunbar SB, Khavjou OA, Bakas T, Hunt G, Kirch RA, Leib AR, Morrison RS, Poehler DC, Roger VL, Whitsel LP: Projected Costs of Informal Caregiving for Cardiovascular Disease: 2015 to 2035: A Policy Statement From the American Heart Association . Circulation 2018,137 (19):e558-e577.

3. Bansilal S, Castellano JM, Fuster V: Global burden of CVD: focus on secondary prevention of cardiovascular disease .International journal of cardiology 2015, 201 Suppl 1 :S1-7.

4. Joseph P, Leong D, McKee M, Anand SS, Schwalm JD, Teo K, Mente A, Yusuf S: Reducing the Global Burden of Cardiovascular Disease, Part 1: The Epidemiology and Risk Factors . Circulation research2017, 121 (6):677-694.

5. Leong DP, Joseph PG, McKee M, Anand SS, Teo KK, Schwalm JD, Yusuf S:Reducing the Global Burden of Cardiovascular Disease, Part 2: Prevention and Treatment of Cardiovascular Disease . Circulation research 2017, 121 (6):695-710. 
6. Hao P, Jiang F, Cheng J, Ma L, Zhang Y, Zhao Y: Traditional Chinese Medicine for Cardiovascular Disease: Evidence and Potential Mechanisms . Journal of the American College of Cardiology 2017,69 (24):2952-2966.

7. Zhao X, Liu Z, Ren Z, Wang H, Wang Z, Zhai J, Cao D, Lyu S, Li L, Lang R et al : Triptolide inhibits pancreatic cancer cell proliferation and migration via down-regulating PLAU based on network pharmacology of Tripterygium wilfordii Hook F . European journal of pharmacology $2020,880: 173225$.

8. Xue M, Jiang ZZ, Wu T, Li J, Zhang L, Zhao Y, Li XJ, Zhang LY, Yang SY: Anti-inflammatory effects and hepatotoxicity of Tripterygium-loaded solid lipid nanoparticles on adjuvant-induced arthritis in rats . Phytomedicine : international journal of phytotherapy and phytopharmacology 2012, 19 (11):998-1006.

9. Choi BS, Sapkota K, Kim S, Lee HJ, Choi HS, Kim SJ:Antioxidant activity and protective effects of Tripterygium regelii extract on hydrogen peroxide-induced injury in human dopaminergic cells, SH-SY5Y . Neurochemical research 2010,35 (8):1269-1280.

10. Peng WH, Chen GL, Zhou Y, Fan SY, Fu DL, Wang Y: Study of expression of circulating inflammatory factors in ACS rats with low dose of Tripterygium Wilfordii . Hellenic journal of cardiology : HJC = Hellenike kardiologike epitheorese 2018, 59 (1):46-47.

11. Li X, Wu N, Zou L, Jia D: Protective effect of celastrol on myocardial ischemia-reperfusion injury . Anatolian journal of cardiology 2017, 18 (6):384-390.

12. Li R, Lu K, Wang Y, Chen M, Zhang F, Shen H, Yao D, Gong K, Zhang Z:Triptolide attenuates pressure overload-induced myocardial remodeling in mice via the inhibition of NLRP3 inflammasome expression . Biochemical and biophysical research communications 2017,485 (1):69-75.

13. Yu H, Shi L, Zhao S, Sun Y, Gao Y, Sun Y, Qi G: Triptolide Attenuates Myocardial Ischemia/Reperfusion Injuries in Rats by Inducing the Activation of Nrf2/HO-1 Defense Pathway . Cardiovascular toxicology 2016, 16 (4):325-335.

14. Lu C, Yu X, Zuo K, Zhang X, Cao C, Xu J, Wang S, Tang T, Ye M, Pei Eet al : Tripterine treatment improves endothelial progenitor cell function via integrin-linked kinase . Cellular physiology and biochemistry : international journal of experimental cellular physiology, biochemistry, and pharmacology 2015,37 (3):1089-1103.

15. Han B, Ge CQ, Zhang HG, Zhou CG, Ji GH, Yang Z, Zhang L:Effects of tripterygium glycosides on restenosis following endovascular treatment . Molecular medicine reports 2016,13 (6):4959-4968.

16. Hopkins AL: Network pharmacology . Nature biotechnology 2007, 25 (10):1110-1111.

17. Lu C, Bing Z, Bi Z, Liu M, Lu T, Xun Y, Wei Z, Yang K:Top-100 Most Cited Publications Concerning Network Pharmacology: A Bibliometric Analysis . Evidence-based complementary and alternative medicine : eCAM 2019, 2019 :1704816.

18. Li P, Chen J, Wang J, Zhou W, Wang X, Li B, Tao W, Wang W, Wang Y, Yang L: Systems pharmacology strategies for drug discovery and combination with applications to cardiovascular diseases . Journal of ethnopharmacology 2014, 151 (1):93-107.

19. Ru J, Li P, Wang J, Zhou W, Li B, Huang C, Li P, Guo Z, Tao W, Yang Y et al : TCMSP: a database of systems pharmacology for drug discovery from herbal medicines. Journal of cheminformatics2014, $6: 13$.

20. Liu F, Li Y, Li M, Wang J, Zhang Y, Du Y, Yang Y: Study on Mechanism of Iridoid Glycosides Derivatives from Fructus Gardeniae in Jiangxi Province by Network Pharmacology . Evidencebased complementary and alternative medicine : eCAM 2020,2020 :4062813. 
21. Huang L, Xie D, Yu Y, Liu H, Shi Y, Shi T, Wen C: TCMID 2.0: a comprehensive resource for TCM . Nucleic acids research 2018,46 (D1):D1117-d1120.

22. Xu J, Wang F, Guo J, Xu C, Cao Y, Fang Z, Wang Q:Pharmacological Mechanisms Underlying the Neuroprotective Effects of Alpinia oxyphylla Miq. on Alzheimer's Disease .International journal of molecular sciences 2020, 21 (6).

23. Daina A, Michielin O, Zoete V: SwissTargetPrediction: updated data and new features for efficient prediction of protein targets of small molecules . Nucleic acids research 2019,47 (W1):W357w364.

24. Wishart DS, Feunang YD, Guo AC, Lo EJ, Marcu A, Grant JR, Sajed T, Johnson D, Li C, Sayeeda Z et al : DrugBank 5.0: a major update to the DrugBank database for 2018 . Nucleic acids research2018, 46 (D1):D1074-d1082.

25. Amberger JS, Hamosh A: Searching Online Mendelian Inheritance in Man (OMIM): A Knowledgebase of Human Genes and Genetic Phenotypes . Current protocols in bioinformatics 2017,58 :1.2.1-1.2.12.

26. Stelzer G, Rosen N, Plaschkes I, Zimmerman S, Twik M, Fishilevich S, Stein TI, Nudel R, Lieder I, Mazor Y et al: The GeneCards Suite: From Gene Data Mining to Disease Genome Sequence Analyses .Current protocols in bioinformatics 2016,54:1.30.31-31.30.33.

27. Szklarczyk D, Franceschini A, Wyder S, Forslund K, Heller D, Huerta-Cepas J, Simonovic M, Roth A, Santos A, Tsafou KP et al :STRING v10: protein-protein interaction networks, integrated over the tree of life . Nucleic acids research 2015,43 (Database issue):D447-452.

28. Blake JA CK, Dolan ME, Drabkin HJ, Hill DP, Ni L, Sitnikov D, Burgess S, Buza T, Gresham C, McCarthy F: Gene Ontology Consortium: going forward . Nucleic acids research 2015,43 (Database issue):D1049-1056.

29. Kanehisa M, Furumichi M, Tanabe M, Sato Y, Morishima K:KEGG: new perspectives on genomes, pathways, diseases and drugs . Nucleic acids research 2017, 45 (D1):D353-d361.

30. Shannon P, Markiel A, Ozier O, Baliga NS, Wang JT, Ramage D, Amin N, Schwikowski B, Ideker T: Cytoscape: a software environment for integrated models of biomolecular interaction networks . Genome research 2003, 13 (11):2498-2504.

31. Li R, Guo C, Li Y, Qin Z, Huang W: Therapeutic targets and signaling mechanisms of vitamin C activity against sepsis: a bioinformatics study . Briefings in bioinformatics 2020.

32. Zhang J, Li H, Zhang Y, Zhao C, Zhu Y, Han M: Uncovering the Pharmacological Mechanism of Stemazole in the Treatment of Neurodegenerative Diseases Based on a Network Pharmacology Approach .International journal of molecular sciences 2020, 21 (2).

33. Song X, Zhang Y, Dai E, Wang L, Du H: Prediction of triptolide targets in rheumatoid arthritis using network pharmacology and molecular docking . International immunopharmacology 2020,80 :106179.

34. Zhou W, Wang Y, Lu A, Zhang G: Systems Pharmacology in Small Molecular Drug Discovery . International journal of molecular sciences 2016, 17 (2):246.

35. Ye S, Luo W, Khan ZA, Wu G, Xuan L, Shan P, Lin K, Chen T, Wang J, Hu X et al : Celastrol Attenuates Angiotensin II-Induced Cardiac Remodeling by Targeting STAT3 . Circulation research2020, 126 (8):1007-1023.

36. Der Sarkissian S, Cailhier JF, Borie M, Stevens LM, Gaboury L, Mansour S, Hamet P, Noiseux N: Celastrol protects ischaemic myocardium through a heat shock response with up-regulation of haeme oxygenase-1 . British journal of pharmacology 2014,171 (23):5265-5279. 
37. Cheng M, Wu G, Song Y, Wang L, Tu L, Zhang L, Zhang C:Celastrol-Induced Suppression of the MiR-21/ERK Signalling Pathway Attenuates Cardiac Fibrosis and Dysfunction . Cellular physiology and biochemistry : international journal of experimental cellular physiology, biochemistry, and pharmacology 2016,38 (5):1928-1938.

38. Liu F, Zhang H, Li Y, Lu X: Nobiletin suppresses oxidative stress and apoptosis in H9c2 cardiomyocytes following hypoxia/reoxygenation injury . European journal of pharmacology2019, $854: 48-53$.

39. Zhang BF, Jiang H, Chen J, Guo X, Li Y, Hu Q, Yang S:Nobiletin ameliorates myocardial ischemia and reperfusion injury by attenuating endoplasmic reticulum stress-associated apoptosis through regulation of the PI3K/AKT signal pathway . International immunopharmacology 2019, 73 :98-107.

40. Guo X, Xue M, Li CJ, Yang W, Wang SS, Ma ZJ, Zhang XN, Wang XY, Zhao R, Chang BC et al : Protective effects of triptolide on TLR4 mediated autoimmune and inflammatory response induced myocardial fibrosis in diabetic cardiomyopathy . Journal of ethnopharmacology 2016, 193 :333-344.

41. Liang Z, Leo S, Wen H, Ouyang M, Jiang W, Yang K: Triptolide improves systolic function and myocardial energy metabolism of diabetic cardiomyopathy in streptozotocin-induced diabetic rats . BMC cardiovascular disorders 2015, $15: 42$.

42. Negrão R, Duarte D, Costa R, Soares R: Isoxanthohumol modulates angiogenesis and inflammation via vascular endothelial growth factor receptor, tumor necrosis factor alpha and nuclear factor kappa B pathways . BioFactors (Oxford, England) 2013,39 (6):608-622.

43. Li C, Liu Y, Xie Z, Lu Q, Luo S: Stigmasterol protects against Ang II-induced proliferation of the A7r5 aortic smooth muscle cell-line. Food \& function 2015, 6 (7):2266-2272.

44. Feng H, Cao J, Zhang G, Wang Y: Kaempferol Attenuates Cardiac Hypertrophy via Regulation of ASK1/MAPK Signaling Pathway and Oxidative Stress . Planta medica 2017, 83 (10):837-845.

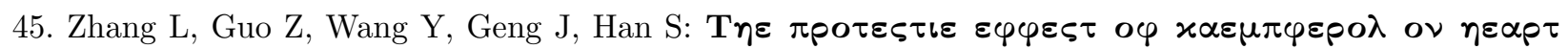

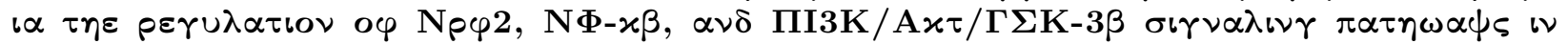

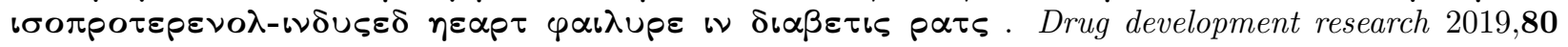
(3):294-309.

46. Abeyrathna $\mathrm{P}, \mathrm{Su} \mathrm{Y}$ : The critical role of Akt in cardiovascular function . Vascular pharmacology 2015,74:38-48.

47. Yu G, Luo Z, Zhou Y, Zhang L, Wu Y, Ding L, Shi Y:Uncovering the pharmacological mechanism of Carthamus tinctorius L. on cardiovascular disease by a systems pharmacology approach .Biomedicine $\& 3$ pharmacotherapy = Biomedecine $\mathscr{E}$ pharmacotherapie2019, $117: 109094$.

48. Leevers SJ, Vanhaesebroeck B, Waterfield MD: Signalling through phosphoinositide 3-kinases: the lipids take centre stage .Current opinion in cell biology 1999, 11 (2):219-225.

49. Carpenter CL, Duckworth BC, Auger KR, Cohen B, Schaffhausen BS, Cantley LC: Purification and characterization of phosphoinositide 3-kinase from rat liver. The Journal of biological chemistry 1990, 265 (32):19704-19711.

50. McLean BA, Patel VB, Zhabyeyev P, Chen X, Basu R, Wang F, Shah S, Vanhaesebroeck B, Oudit

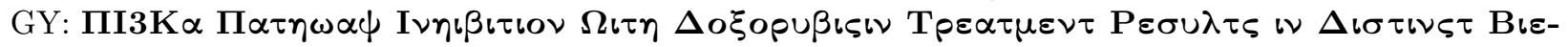

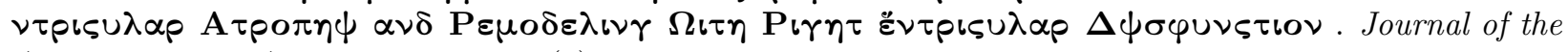
American Heart Association 2019, 8 (9):e010961. 
51. Network CGA: Comprehensive molecular portraits of human breast tumours . Nature 2012, 490 (7418):61-70.

52. Pearson G, Robinson F, Beers Gibson T, Xu BE, Karandikar M, Berman K, Cobb MH: Mitogenactivated protein (MAP) kinase pathways: regulation and physiological functions . Endocrine reviews 2001,22 (2):153-183.

53. Wang Y, Zhang X, Gao L, Li J, Chen W, Chi J, Zhang X, Fu Y, Zhao M, Liu N et al : Cortistatin exerts antiproliferation and antimigration effects in vascular smooth muscle cells stimulated by Ang II through suppressing ERK1/2, p38 MAPK, JNK and ERK5 signaling pathways . Annals of translational medicine 2019,7 (20):561.

54. Taniyama Y, Ushio-Fukai M, Hitomi H, Rocic P, Kingsley MJ, Pfahnl C, Weber DS, Alexander RW, Griendling KK: Role of p38 MAPK and MAPKAPK-2 in angiotensin II-induced Akt activation in vascular smooth muscle cells . American journal of physiology Cell physiology2004, 287 (2):C494-499.

55. Kyotani Y, Zhao J, Tomita S, Nakayama H, Isosaki M, Uno M, Yoshizumi M: Olmesartan inhibits angiotensin II-Induced migration of vascular smooth muscle cells through Src and mitogenactivated protein kinase pathways . Journal of pharmacological sciences 2010,113 (2):161-168.

56. Visconte C, Canino J, Guidetti GF, Zarà M, Seppi C, Abubaker AA, Pula G, Torti M, Canobbio I: Amyloid precursor protein is required for in vitro platelet adhesion to amyloid peptides and potentiation of thrombus formation . Cellular signalling 2018,52 :95-102.

57. Canobbio I, Visconte C, Momi S, Guidetti GF, Zarà M, Canino J, Falcinelli E, Gresele P, Torti M: Platelet amyloid precursor protein is a modulator of venous thromboembolism in mice . Blood 2017 , 130 (4):527-536.

58. Kolovou V, Tsipis A, Mihas C, Katsiki N, Vartela V, Koutelou M, Manolopoulou D, Leondiadis E, Iakovou I, Mavrogieni S et al :Tumor Protein p53 (TP53) Gene and Left Main Coronary Artery Disease . Angiology 2018, 69 (8):730-735.

59. Tiwari S, Dwivedi UN: Discovering Innovative Drugs Targeting Both Cancer and Cardiovascular Disease by Shared Protein-Protein Interaction Network Analyses . Omics : a journal of integrative biology 2019, 23 (9):417-425.

60. Ivanov AA, Khuri FR, Fu H: Targeting protein-protein interactions as an anticancer strategy . Trends in pharmacological sciences 2013, 34 (7):393-400.

61. Sarajlić A, Janjić V, Stojković N, Radak D, Pržulj N:Network topology reveals key cardiovascular disease genes .PloS one 2013, 8 (8):e71537.

62. Zhang C, He XJ, Li L, Lu C, Lu AP: Effect of the Natural Product Triptolide on Pancreatic Cancer: A Systematic Review of Preclinical Studies . Frontiers in pharmacology 2017,8:490.

63. Wen L, Chen Y, Zeng LL, Zhao F, Yi S, Yang LJ, Zhang BP, Zhao J, Zhao ZC, Zhang C: Triptolide Induces Cell Apoptosis by Targeting H3K4me3 and Downstream Effector Proteins in KM3 Multiple Myeloma Cells .Current pharmaceutical biotechnology 2015,17 (2):147-160.

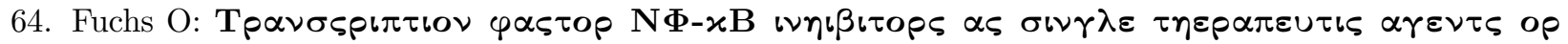

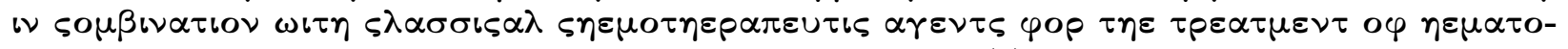

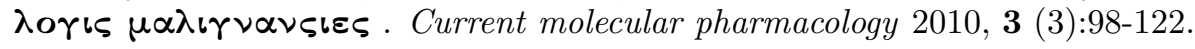

Hosted file

Table 1.pdf available at https://authorea.com/users/362663/articles/483729-networkpharmacology-based-analysis-of-the-mechanisms-of-tripterygium-wilfordii-hook-f-oncardiovascular-disease 


\section{Hosted file}

Supplementary Table.xlsx available at https://authorea.com/users/362663/articles/483729network-pharmacology-based-analysis-of-the-mechanisms-of-tripterygium-wilfordii-hook-fon-cardiovascular-disease

\section{Hosted file}

Figure 1.pdf available at https://authorea.com/users/362663/articles/483729-networkpharmacology-based-analysis-of-the-mechanisms-of-tripterygium-wilfordii-hook-f-oncardiovascular-disease

\section{Hosted file}

Figure 2.pdf available at https://authorea.com/users/362663/articles/483729-networkpharmacology-based-analysis-of-the-mechanisms-of-tripterygium-wilfordii-hook-f-oncardiovascular-disease

\section{Hosted file}

Figure 3.pdf available at https://authorea.com/users/362663/articles/483729-networkpharmacology-based-analysis-of-the-mechanisms-of-tripterygium-wilfordii-hook-f-oncardiovascular-disease

\section{Hosted file}

Figure 4.pdf available at https://authorea.com/users/362663/articles/483729-networkpharmacology-based-analysis-of-the-mechanisms-of-tripterygium-wilfordii-hook-f-oncardiovascular-disease

\section{Hosted file}

Figure 5.pdf available at https://authorea.com/users/362663/articles/483729-networkpharmacology-based-analysis-of-the-mechanisms-of-tripterygium-wilfordii-hook-f-oncardiovascular-disease

\section{Hosted file}

Figure 6.pdf available at https://authorea.com/users/362663/articles/483729-networkpharmacology-based-analysis-of-the-mechanisms-of-tripterygium-wilfordii-hook-f-oncardiovascular-disease 